Treatment of the latter with cadmium chloride yields the cadmium chloride complex of crude lecithin. Presumably, then, the above reactions have taken place in the absence of both cardiolipin and cholesterol. However, the possibility of there still being a small amount of cardiolipin present has been considered, and repeat experiments on similar lines are in progress using both partially purified and purified lecithin solutions.

Other precipitates from alcoholic extracts of beef heart which have been tested are those produced by treatment of the extracts with 1 per cent sodium gold chloride and 5 per cent silver nitrate solutions respectively.

The precipitate formed with the sodium $g$ ld chloride solution is bright orange-yellow. Suspension of this material in a phosphate buffer solution of $p \mathrm{H} 6 \cdot 4$ yields a yellowish colloidal solution which when tested with syphilitic serum gives only a feeble reaction, although normal sera are clearly negative. However, on exposure of the colloidal solution to light for fifteen minutes, a ruby red coloration develops similar to that noted in Lange's colloidal gold solution. This 'colloidal gold' suspension is strongly active with syphilitic sera, giving a bronze precipitate in an almost colourless fluid; but an adjustment of the $p H$ is considered necessary for the production of more highly specific results. It is to be noted that the ruby red colour fails to develop when the solution is stored in the dark either at room temperature or in the refrigerator.

The silver nitrate precipitates formed with alcoholic extracts assume a brown colloidal state when suitably suspended. These react strongly with syphilitic sera, the positive precipitate being dark brown, while a negative reaction is shown by a light-brownish opalescent solution.

An adequate number of tests have not so far been made to determine the actual specificity and sensitivity of the reactions obtained with these precipitates.

Further details of the work in progress will be given at a later date, together with the results obtained with precipitates formed by treatment of both crude and purified extracts of the apparently active fractions of beef heart muscle with the salts of calcium, uranium, lead and mercury. Comparative readings will be made with diagnostic antigens such as are used in the Eagle flocculation, Kahn precipitation and Kolmer complement-fixation procedures.

GWen M. Macnab

South African Institute for Medical Research, Johannesburg.

Feb. 24 .

\section{Magnetic Susceptibilities of Polyindene Fractions}

Farquharson and $\mathrm{Ady}^{1}$ examined the polymerization of 2,3-dimethylbutadiene, and Bhatnagar, Kapur and $\mathrm{Kaur}^{2}$ that of styrene by measuring the magnetic susceptibilities. But there are no determinations comparing the susceptibilities at different degrees of polymerization.

This has been done for polyindene fractions by the cylinder method, resulting in a kinetic investigation ${ }^{3}$. The field strength of the magnet used was calibrated by chloroform $\left(\chi=-0.61 \times 10^{-6}\right)$ and amounted to 9,460 oersteds with poles $7 \cdot 1 \mathrm{~mm}$. apart and at $7 \mathrm{amp} .\left(\chi_{B}\right.$ is the susceptibility of a basic molecule).

$\begin{array}{rcc}\text { Fraction } & \text { Degree of polymerization } & -\chi_{B} \times 10^{6} \\ \text { I } & 2 \mathbf{4 5} & 81 \cdot 7 \\ \text { II } & 2 \cdot 56 & 85 \cdot 5 \\ \text { III } & 3 \cdot 59 & 90 \cdot 6 \\ \text { II } & 3 \cdot 92 & 90 \cdot 8 \\ \text { VI } & 4 \cdot 21 & 93 \cdot 8 \\ \text { VII } & 4 \cdot 65 & 95 \cdot 3 \\ \text { VII } & 5 \cdot 36 & 96 \cdot 1 \\ \text { III } & 5 \cdot 74 & 92 \cdot 1 \\ \text { IX } & 7 \cdot 39 & 80 \cdot 4\end{array}$

The plot of the diamagnetic susceptibility against degree of polymerization (see graph) shows a maximum at the original medium degree of polymeriza. tion; the stability of the latter is therefore marked by its magnetic behaviour too. The rise is explicable by the decrease in double links with increasing degree of polymerization, the double links possessing a paramagnetic increment (Pascal). At higher degrees of polymerization, a paramagnetic component seems to become of increasing importance.

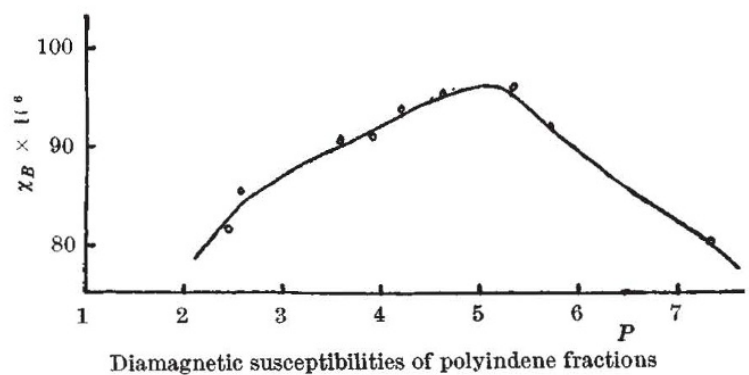

Further experiments will show whether the effect is of general significance or not.

Institute of Physical Chemistry,

W. SCHÜTZNER

Technical University, Vienna.

1 Trans. Farad. Soc., 32, 219 (1936); Nature, 143, 1067 (1939). ${ }^{2} J$. Ind. Chem. Soc., 17, 177.

a "Polymer Science", 3, No. 4, 535 (1948).

\section{Globule Size in Two-Phase Emulsions}

A THEORY that in an oil hydrosol the globules tend to reach a stable size, at which there is a balance between electrical and capillary forces, was advanced by Lewis ${ }^{1}$ and extended by Knapp ${ }^{2}$ for solid particles. For a particle surrounded by an electrical double layer, Knapp derived the equation

$$
r_{c}=+\left(\frac{e^{2} \delta}{8 \pi \varepsilon \gamma}\right)^{1 / 4},
$$

where $r_{c}$ is the 'critical' radius at which the solubility of the particle is equal to that of a plane surface of the same material, $e$ is the electric charge on the particle, $\delta$ the thickness of the double layer (assumed to be small compared with $r_{c}$ ), $\varepsilon$ the dielectric constant of the medium and $\gamma$ the interfacial tension between particle and medium.

Lewis $^{3}$ considered in some detail the process of growth of an oil globule by the accretion of uncharged oil molecules to a charged ultramicron. He deduced that, in consequence of equation (1), the ultimate charge density should be independent of the globule radius, and argued that the size eventually reached by an ultramicron should be determined by its initial charge, which was assumed to remain constant during growth. 